\title{
1946 l'année charnière, le passage d'une société technique à une société savante
}

\author{
Elodie Massol ${ }^{\mathrm{a}}$
}

Reçu le 14 novembre 2019 / Accepté le 19 novembre 2019

\begin{abstract}
Résumé - 1946 fut une année clef pour la Société Hydrotechnique de France. La nationalisation de l'énergie fut pour l'association source de nombreux défis. Nous allons nous intéresser à cette période historique et essayer de comprendre comment la SHF a su s'adapter à cette nouvelle donne et à se réinventer pour garder son statut de référence dans le domaine hydrotechnique français.
\end{abstract}

Mots clés : SHF / EDF / nationalisation / 1946 / hydroélectricité

\begin{abstract}
- 1946 a milestone year, the change from a technical company to a learned society. 1946 was a key year for the "Société Hydrotechnique de France". The nationalisation of the energy was the root of numerous challenges. We will focus on this historical period and try to understand how the SHF successfully adapted to this new order and reinvented itself to keep its reference position in the french hydrotechnical area.
\end{abstract}

Keywords: SHF / EDF / nationalisation / 1946 / hydroelectricity

\section{La SHF, innovation et héritage}

En 2019, la Société Hydrotechnique de France (SHF) fête ses 107 ans d'existence. Un siècle de connaissance approfondie des savoir-faire et des techniques du monde hydraulique. Depuis sa création en 1912 pour étudier la question de l'aménagement et de l'exploitation des chutes d'eau à la force actuelle de sa communauté scientifique internationale, la SHF a connu des mutations et des évolutions qui lui ont permis de développer un savoir-faire propre. Tout en égrainant le $\mathrm{XX}^{\mathrm{e}}$ siècle, nous allons nous pencher sur la consistance même de cette société savante.

Dès la fin du $\mathrm{XIX}^{\mathrm{e}}$ siècle les pays occidentaux connaissent une deuxième industrialisation consécutive à la révolution électrique. La France accuse un retard dû à un manque de capitaux. C'est le premier conflit mondial qui va créer une prise de conscience de la dépendance hexagonale au charbon. Il va être suivi par une réelle volonté politique d'indépendance

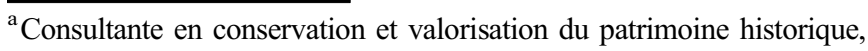
France. énergétique. Avant 1914, l'installation hydroélectrique la plus puissante était celle de l'Argentière sur la Durance avec $28 \mathrm{MW}$, créée en 1909, alors première au niveau européen. Mais c'est une révolution hydraulique qui va avoir lieu dans l'entre-deux-guerres, lancée dès le 16 octobre 1919 par la loi relative à l'utilisation de l'énergie hydraulique, qui instaure sa nationalisation. Une réelle mise en valeur du potentiel hydroélectrique de la France est alors possible. Des centrales de plus grande taille voient le jour. On peut citer Eguzon (53 MW) dans l'Indre en 1926, Sarrans (167 MW) dans l'Aveyron en 1932, Kembs (160 MW) sur le Rhin en 1932, Sautet (74 MW) sur le Drac en 1934 ou Marèges (140 MW) sur la Dordogne en 1935.

\section{1 État des lieux de la SHF en 1946}

La Société Hydrotechnique de France (SHF), créée en 1912, rassemble alors l'ensemble des personnalités concernées par le développement et l'aménagement des équipements hydroélectriques. Mi-société savante, mi-société technique, elle est alors considérée comme l'organe technique de la Chambre Syndicale des Forces Hydrauliques. Interlocuteur privilégié aussi bien du gouvernement, comme en 1917 dans le cadre des grandes réflexions sur la dépendance trop forte du pays à l'énergie charbonnière, mais aussi des sociétés techniques, en produisant un grand nombre de cahiers des charges visant à résoudre les problèmes rencontrés par ces dernières dans l'exploitation de la houille blanche. Les années 
1920 sont marquées par l'ouverture de ses propres laboratoires de recherche et la création en 1927 d'un service d'essais et de contrôle permanent des installations hydrauliques.

$\mathrm{Au}$ début des années quarante la SHF connaît une importante phase de croissance et ce malgré les difficultés engendrées par le second conflit mondial. 1941 fut une année charnière, la SHF décide de réformer son organisation afin de mieux s'adapter aux nouvelles réalités du secteur hydrotechnique. Elle créée un service d'études générale de statistiques et d'hydrologie qui accomplit un important travail de documentation. Ce service donne lieu à la première publication des Annuaires hydrologiques de la France, reprenant ainsi le flambeau du ministère de l'agriculture (1911-1924). Elle décide également de revoir ses statuts, son conseil d'administration souhaitant laisser une plus grande place, en son sein, aux personnalités «ayant joué récemment un rôle important dans le domaine des équipements hydroélectriques ». Une désignation réservée à des membres d'honneur est ainsi créée. En outre, est instauré un comité de liaison chargé d'assurer la coordination nécessaire entre les industriels présents au sein du conseil d'administration de l'association et les personnalités scientifiques qui composent le comité technique. «Il a pour tâche d'orienter l'activité des services de l'association dans le sens des besoins de l'industrie en leur permettant de bénéficier des études et des conseils du comité technique ». Ce comité permet alors à la SHF d'adapter son action à la croissance et à l'ambition du domaine hydroélectrique français. Ces évolutions viennent confirmer la volonté de la SHF d'être un acteur de terrain, œuvrant au plus près des questions techniques et des industriels du domaine. Mais le second conflit mondial et le monde nouveau qui en ressort vont mettre à mal cette volonté et lancer un nouveau défi à l'association, celui de se réinventer.

Au sortir de la guerre l'action de la SHF s'organise autour de 4 grands domaines d'activité :

Premièrement son service d'hydrologie statistique et d'études générales qui, comme nous l'avons évoqué, publie depuis 1941 les Annuaires hydrologiques de la France. Des données constamment enrichies, ainsi en 1946 se voient rajoutés les débits moyens mensuels, des courbes de probabilité et des prévisions de débits.

Son service d'essais, de réception et de contrôle fut impacté par le second conflit mondial, du fait des difficiles conditions de fonctionnement des usines hydrotechniques, mais aussi les difficultés de transport du matériel et du personnel. Il faudra attendre 1946 pour qu'il reprenne une activité normale. Ce service opère dans les usines hydrotechniques, voire sur les rivières. Il effectue un grand nombre d'essais de contrôles des rendements ou de réception. À la veille de son basculement au sein du nouvel établissement public, le service envisageait de mettre en application la méthode Gibson pour la mesure du débit d'une conduite forcée. Concernant ses actions en rivière, il procède à de réguliers jaugeages et tarages d'échelles limnimétriques.

Son action s'exprime également dans le domaine de la recherche au travers de ses laboratoires. Celui de Beauvert, près de Grenoble, créé en 1922 vient alors d'être fermé en date du 31 juillet 1945 et celui du Saulcy à Metz, créé en 1929, semble avoir fermé quelques temps auparavant. Aussi le 12 avril 1945, la SHF récupère la concession du laboratoire de Chatou en Ile de France, projet imaginé par le Service de la navigation dès 1939. Elle est chargée à titre précaire et révocable de l'aménager et de l'exploiter. On constate au mois de juin 1946 une avancée certaines des travaux, dont la fin est prévue pour l'année suivante. La SHF continue aussi son travail de recherche dans ses usines partenaires où elle mène un certain nombre d'essais techniques. On peut citer pour cette année 1946, des essais sur modèles pour l'étude de l'agrandissement des chambres d'eau des usines de Nouaux et de Lamativie.

Dernier aspect, le rôle de son comité technique qui coordonne et gère la publication des résultats de l'ensemble des études techniques relatives à l'objet de l'association. Complété depuis 1941 par un comité de liaison.

\section{Nationalisation de l'énergie et réorganisation de l'association}

La création d'électricité de France (EDF) le 8 avril 1946, par la loi n ${ }^{\circ} 46-628$ sur la nationalisation de l'électricité et du gaz, va venir modifier en profondeur le fonctionnement et les domaines d'intervention de la SHF. Ce projet de loi, profondément inspiré des idées duConseil National de la Résistance (CNR), a contribué à nationaliser 1450 entreprises de production, de transport et de distribution d'énergie électrique. Le CNR, sous l'impulsion de Pierre Villon, décide de reconstruire la nation en suivant les principes communistes de nationalisation et d'économie planifiée. Entre autres "l'indépendance de la presse à l'égard de l'État et des puissances d'argent; l'égalité absolue de tous les citoyens devant la loi; le retour à la nation des grands moyens de production monopolisée, fruits du travail commun, des sources d'énergie, des richesses du sous-sol, des compagnies d'assurance et des grandes banques ; la garantie d'un niveau de salaire et de traitement qui assure à chaque travailleur et à sa famille la sécurité, la dignité et la possibilité d'une vie pleinement humaine; un plan complet de sécurité sociale...». C'est en s'inspirant de ce programme que le ministre de la production industrielle Marcel Paul (PCF) déclare, dès sa nomination en 1945 , vouloir « agir efficacement au maintien, au renforcement de l'union de toutes les forces patriotiques, à l'union entre eux des groupements de la Résistance, à la réalisation de la grande mobilisation en faveur et pour l'application du programme du CNR. Il faut gagner la bataille de la nationalisation de l'électricité et du gaz».

C'est chose faite dès le 8 avril 1946 et de fait l'action de la SHF s'en voit modifiée en profondeur. En effet, il est alors décidé que l'association ne conserve que ses activités de société savante et qu'elle transfère à EDF la gestion de ses services techniques. La fin de l'année 1946 est alors consacrée à un état des lieux de ces derniers et à l'établissement des modalités de transfert. Ainsi dans un projet de lettre adressé par le Président de la SHF, Henri Milon, au Président directeur général d'électricité de France, Pierre Simon, en date du 24 octobre 1946, le transfert est envisagé comme suit:

Premièrement EDF va devoir, à une date non déterminée à l'écriture du courrier, prendre en charge «la totalité du personnel des services techniques de [l'] association, à savoir le laboratoire de Chatou, le service d'essais et du contrôle permanent et le service hydrologique». Concernant les transferts matériels, la SHF est alors encore propriétaire des immeubles et de l'équipement du laboratoire de Beauvert, 
fermé l'année précédente, mais également du matériel du service d'essais et du mobilier de son siège social incluant une bibliothèque. Il est décidé qu'elle conserve son siège social et son mobilier rue de Grenelle, pour le reste des biens il est proposé une mise à disposition de la nouvelle société nationalisée sous la forme d'une location-vente prévue sur 25 ans. Cette location est proposée au prix de 500000 francs annuels, qui sera finalement réévalué et ratifié à 550000 francs, dont 250000 pour la vente des bâtiments et 300000 d'indemnisation pour résiliation du bail qui avait été consenti par la Société des ateliers Meyret-Beylier et Piccard-Pictet à l'association le 13 mars 1919. Le petit matériel est lui remis gracieusement à la nouvelle société. Concernant le projet du laboratoire de Chatou, tout juste démarré, la SHF propose alors que le transfert de ses activités techniques prévoit également une substitution d'EDF à la SHF dans son rôle d'exploitant. Il deviendra alors le laboratoire national d'hydraulique de Chatou. Le président de la SHF propose à son homologue que soient actés l'ensemble de ces transferts au $1^{\mathrm{er}}$ novembre suivant. Cette cession des biens est finalement ratifiée le 11 février 1947.

\section{Une nouvelle SHF}

La SHF se voit alors définitivement coupée de ses activités techniques et se concentre sur ses activités de recherche et ses publications. Malgré l'intégration de son service hydrologique au sein de la nouvelle société nationale, elle conserve l'édition des Annuaires hydrologiques de la France et ce jusqu'en 1969 et sa reprise par le ministère de l'agriculture. S'opère alors une nouvelle modification de ses statuts qui entérine l'ensemble de ces changements lors de l'assemblée générale extraordinaire du 27 mars 1947. Les nouveaux statuts de l'association reflètent cette nouvelle orientation, son objet principal étant désormais "l'étude des toutes questions relatives à l'hydraulique, à la mécanique des fluides et aux sciences qui s'y rattachent ». La séparant ainsi de toute préoccupation d'ordre industriel. Son comité technique s'est étoffé dans les années qui ont suivies. De trente membres au plus à l'origine, il en compte aujourd'hui plus de 600 venus d'univers variés «hommes de laboratoires, hommes de chantiers, constructeurs de turbines et de conduites, entrepreneurs, fonctionnaires, jeunes ingénieurs côtoyant les plus éminents de leurs aînés ». Il se réorganise également, pour favoriser l'échange scientifique et technique entre ses membres et la diffusion des connaissances, en organisant des cessions tri annuelles, en mars, juin et novembre, et des congrès biennaux, appelés Journées de l'Hydraulique. Ces cessions furent pour beaucoup de jeunes chercheurs un banc d'essai pour présenter leurs travaux, face à un impressionnant auditoire de scientifiques chevronnés. Ces interventions donnaient lieu à des comptes rendus qui paraissaient dans la revue de la SHF, Mémoires et Travaux, jusqu'en 1965, puis par la suite dans La Houille Blanche. La participation à ces sessions était gratuite, elle l'est restée jusqu'aux années 1980, grâce au mécénat très efficace d'EDF. Les Journées de l'Hydraulique ont quant à elles connu une première édition du 21 au 23 juin 1949, initiées par le président d'alors Henri Milon. Elles sont aujourd'hui décrites par les membres de l'association comme: «de grandes kermesses scientifiques de l'eau avec très souvent une participation internationale notoire $»$.

La nouvelle vie de la SHF débutée après-guerre fut des plus intense. Face à la perte d'une grande partie ses actifs et de ses services, elle a su se réinventer et donner une nouvelle dimension à ses activités. De 1960 aux années 2000 «la SHF, ce fut 98 manifestations en 40 ans ! Soit environ 2,5 réunions par an». Un rythme soutenu qui fut même supérieur à la moyenne de 2 réunions annuelles du comité technique observée entre 1912 et 1955 . La SHF c'est également trois prix de recherche remis annuellement. Elle a su se réinventer, renforçant sa légitimité en tant que société savante, s'adaptant sans cesse aux évolutions sociétales et aux besoins du domaine. On peut citer par exemple la prise en compte des questions humaines et sociales liées à la ressource en eau. Comme la décrivent ses membres «la SHF est un lieu privilégié d'échanges scientifiques et culturels entre générations mêlant l'humour et l'indulgence ». La SHF avec son écho La Houille Blanche a été et reste l'ambassadeur de la France dans le domaine de l'eau.

EDF et la SHF ont su avancer en se soutenant mutuellement et la volonté de préserver ce lien fut claire dès les années 1950. Pierre Massé, directeur général adjoint de la société nationale de de 1949 à 1969, prend la présidence de la SHF de 1952 à 1953. En 2013 la SHF rendra hommage à ce grand homme en donnant son nom au dernier-né de ses prix scientifiques dédié aux études en sciences humaines et sociales, appliquées au domaine de l'eau.

\section{Fonds documentaire}

Anonyme. Note (La SHF et son domaine d'activité), 15 mai 1946 (archives de la SHF).

Banal M, Perrin M, Thirriot C. 2002. Histoire de la SHF. La Houille Blanche 4(5): 15-19.

Bordes J-L. 2010. Les barrages en France du XVIII ${ }^{\mathrm{e}}$ à la fin du $\mathrm{XX}^{\mathrm{e}}$ siècle. «Pour mémoire» 9: 70-120.

Le Gros C, Sauquet E, Lang M, Achard A-L, Leblois E, Biton B. 2015. Les annuaires hydrologiques de la Société Hydrotechnique de France : une source d'information patrimoniale pour la connaissance de l'hydrologie en France. La Houille Blanche 4: 66-77.

Milon H. 1946. Projet de lettre adressée à M. Pierre Simon, Président directeur général d'électricité de France, 24 octobre 1946 (archives de la SHF).

Remenieras G. 1946. Quelques renseignement sur l'activité des services techniques de la Société Hydrotechnique de France, 29 juin 1946 (archives de la SHF).

SHF. Assemblée constitutive (procès-verbal), 26 mars 1912 (archives de la SHF).

SHF. Assemblée générale extraordinaire (procès-verbal), 22 janvier 1941 (archives de la SHF).

SHF. Assemblée générale extraordinaire (procès-verbal), 27 mars 1947 (archives de la SHF).

SHF. 1949. Journées de l'hydraulique. La Houille Blanche numéro spécial B: 673-687.

Wolikoff S. 2004. L'esprit de la Résistance toujours d'actualité. Le Monde diplomatique 26. 